\title{
Relativistic plasma and ICM/radio source interaction
}

\author{
Luigina Feretti ${ }^{1}$, Gabriele Giovannini ${ }^{1,2}$, Federica Govoni ${ }^{3}$, and \\ Matteo Murgia ${ }^{3}$ \\ ${ }^{1}$ INAF Istituto di Radioastronomia, \\ Via P. Gobetti n. 101, 40129 Bologna, Italy \\ email: lferetti@ira.inaf.it \\ ${ }^{2}$ Dipartimento di Astronomia, Universitá di Bologna, \\ via Ranzani n.1, 40127 Bologna, Italy \\ ${ }^{3}$ INAF Osservatorio Astronomico di Cagliari, \\ Strada 54, Loc. Poggio dei Pini, 09012 Capoterra, Italy
}

\begin{abstract}
The first detection of a diffuse radio source in a cluster of galaxies, dates back to the 1959 (Coma Cluster, Large et al. 1959). Since then, synchrotron radiating radio sources have been found in several clusters, and represent an important cluster component which is linked to the thermal gas. Such sources indicate the existence of large scale magnetic fields and of a population of relativistic electrons in the cluster volume. The observational results provide evidence that these phenomena are related to turbulence and shock-structures in the intergalactic medium, thus playing a major role in the evolution of the large scale structure in the Universe. The interaction between radio sources and cluster gas is well established in particular at the center of cooling core clusters, where feedback from AGN is a necessary ingredient to adequately describe the formation and evolution of galaxies and host clusters.
\end{abstract}

Keywords. galaxies: clusters: general, cooling flows, intergalactic medium, magnetic fields, radio continuum: general, radiation mechanisms: nonthermal

\section{Introduction}

Clusters of galaxies are the largest gravitationally bound systems in the Universe. Most of the gravitating matter in any cluster is in the form of dark matter $(\sim 80 \%)$. Some of the luminous matter is in galaxies $(\sim 3-5 \%)$, the rest is in diffuse hot gas $(\sim 15-17 \%)$, detected in X-ray through its thermal bremsstrahlung emission. This thermal plasma, consisting of particles of energies of several $\mathrm{keV}$, is commonly referred to as Intracluster Medium (ICM). In recent years it has become clear that the ICM can also contain highly relativistic particles, whose number density is of the order of $10^{-10} \mathrm{~cm}^{-3}$. Although the relativistic plasma has an energy density of $<1 \%$ than that of the thermal gas, it is nevertheless very important in the cluster formation and evolution.

Clusters are formed by hierarchical structure formation processes. In this scenario, smaller units formed first and merged to larger and larger units in the course of time. The merger activity appears to be continuing at the present time, and explains the relative abundance of substructure and temperature gradients detected in Abell clusters by optical and X-ray observations. At the end of their evolution, clusters reach a relaxed state, with a giant galaxy at the center, and enhanced X-ray surface brightness peak in the cores. The hot gas in the centre has a radiative cooling time shorter than the expected cluster age, therefore energy losses due to X-ray emission are important and lead to a temperature drop towards the centre (Fabian 1994). The relaxed clusters are thus referred to as cooling core clusters. 
From the radio point of view, clusters can host diffuse radio emission, which has been now revealed in several conditions (merging and relaxed clusters), at different cluster locations (center, periphery, intermediate distance), and on very different size scales (100 kpc to $>\mathrm{Mpc}$ ),(see Fig. 1 for several examples). All diffuse radio sources have in common the short lifetimes of the radiating particles, which therefore need to be reaccelerated. The properties of the radio emission are linked to those of the host cluster, therefore the connection between the thermal and relativstic plasma in clusters of galaxies is important for the cluster formation and evolution. The understanding of magnetic field and relativistic particle properties is important for a comprehensive physical description of the intracluster medium in galaxy clusters.
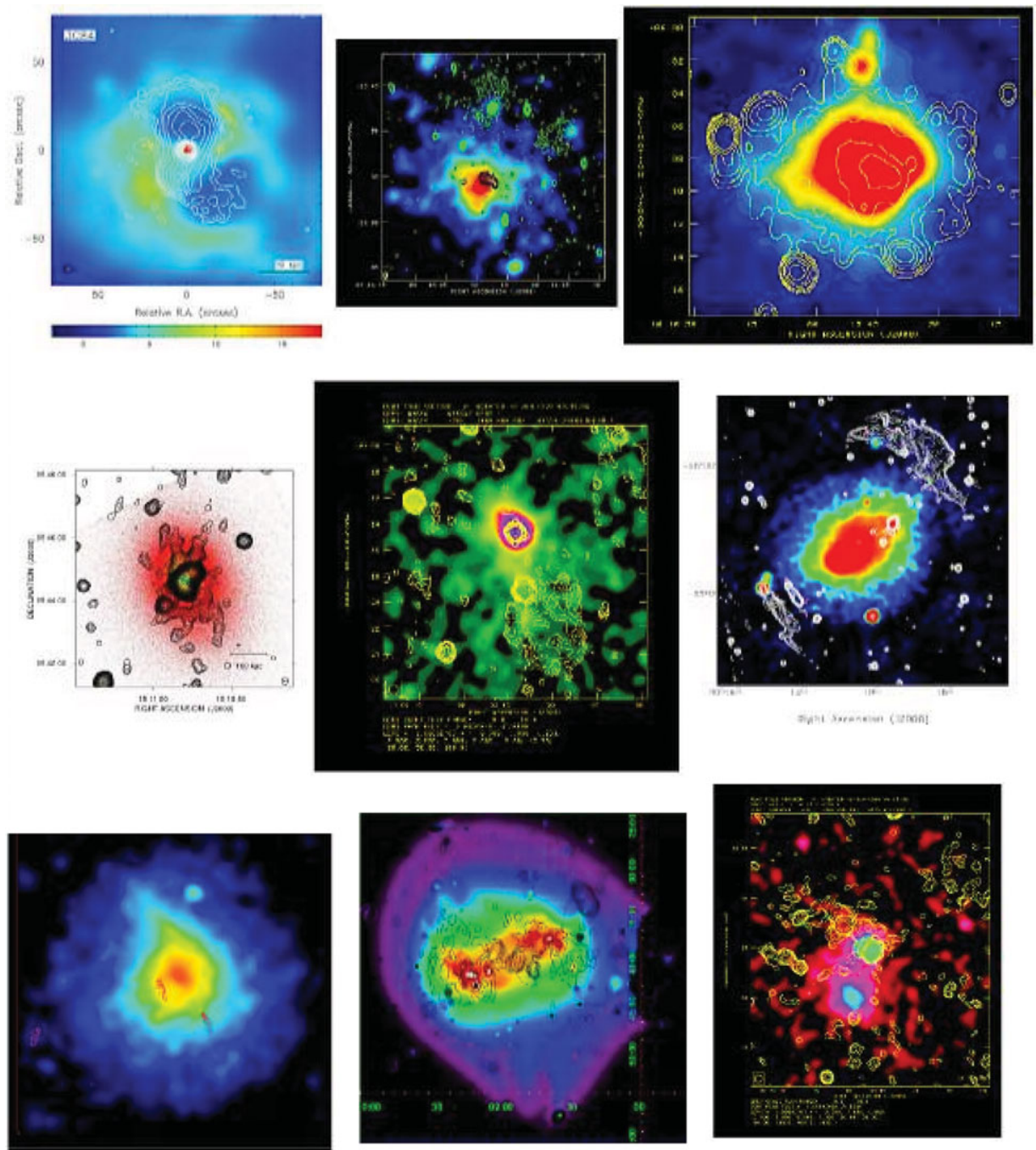

Figure 1. Collection of clusters showing several types of radio emission, shown in contours, overlaid onto the X-ray emission, shown in colors. Clusters are (from left to right and from top to bottom) Perseus (mini-halo and X-ray cavity), A 548b (relic), A 2163 (halo), A 2029 (mini-halo), A 1664 (relic), A 3667 (double relics), A 119 (radio galaxies), A 754 (halo plus relic), A 115 (relic). 


\section{Diffuse cluster radio sources in merging clusters}

The most spectacular example of diffuse cluster radio sources is represented by giant radio halos. They are associated with clusters undergoing merging processes, and believed to be energized by the turbulence produced during the cluster mergers (see Feretti \& Giovannini 2008 for a review). Radio halos (Fig. 2) can reach a size of $1-2 \mathrm{Mpc}$ and more, although smaller size halos have also been found. New halos have recently been detected in A 851, A 1213, A 1351, A 1995, A 2034 and A 2294 (Giovannini et al. 2009, also Giacintucci et al. 2009 for A 1351). The most powerful radio halo known so far is found in the distant cluster MACS J0717.5 +3745 (Bonafede et al. 2009b, van Weeren et al. 2009) at $\mathrm{z}=0.55$. A peculiar example of a double radio halo in a close pair of galaxy clusters is represented by A 399 and A 401 (Murgia et al. 2010), where the two radio halos could be either originated by previous merger histories of the two clusters, or due to the currently ongoing interaction.

The radio power of both small and giant halos correlates with the cluster X-ray luminosity, i.e. gas temperature and total mass (Cassano et al. 2006, Giovannini et al. 2009), in the sense that highly luminous X-ray clusters host the most powerful radio halos. The radio halos are generally associated with clusters with X-ray luminosity in the 0.1-2.4 keV range $>>10^{44} \mathrm{erg} \mathrm{s}^{-1}$. Recently, however, radio halos have been found also in clusters with X-ray luminosity around $10^{43} \mathrm{erg} \mathrm{s}^{-1}$, which are typical of low density environments: the first example is the cluster A 1213 (Giovannini et al. 2009), other cases are presented by Brown \& Rudnick 2009.

Another important link between the relativistic and thermal plasma is represented by the connection between the cluster temperature and the radio halo spectral index, first suggested by Feretti et al. 2004, and now confirmed by Giovannini et al. 2009.
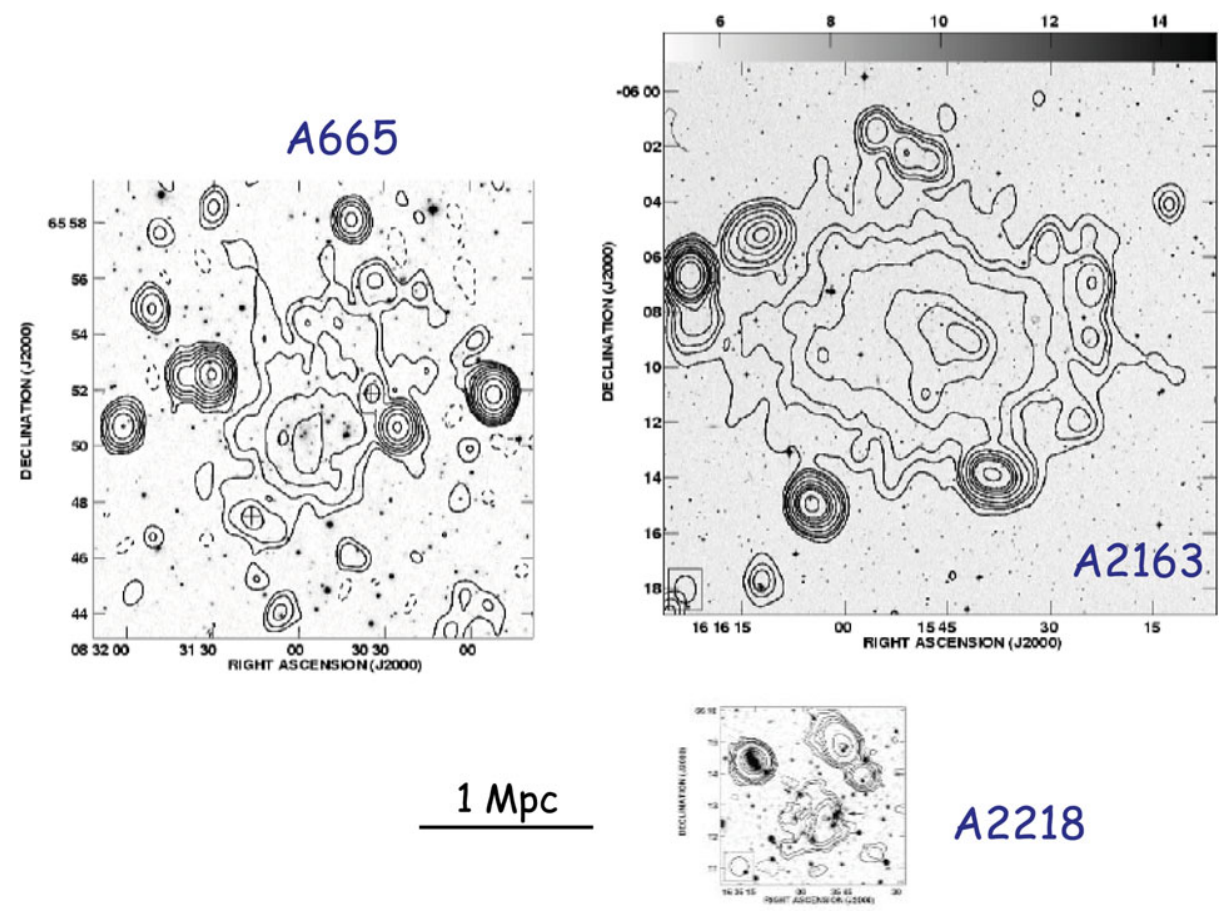

Figure 2. Images of the clusters A 665, A 2163 and A 2218, hosting radio halos: radio emission is represented by contours, which are overlaid onto the optical image. The maps are all scaled to the same linear scale. 
It is found that clusters at higher temperature tend to host halos with flatter spectra (see Fig. 3). This correlation reinforces the connection between radio emission and cluster mergers, since hot clusters are more massive and may derive from more energetic merging processes, supplying more energy to the radiating electrons.

Other diffuse radio sources associated with cluster mergers are relics, located in cluster peripheral regions, and characterized by high polarized emission (see Giovannini \& Feretti 2004 for a review and references). Relics can be also detected in clusters containing radio halos. A very narrow giant relic, which displays highly aligned magnetic field, has been recently detected by van Weeren et al. 2010. Remarkable are the giant double relics, located on opposite sides with respect to the cluster center, whose prototype is A 3667 (Röttgering et al. 1997). Several other cases have been found more recently: RXCJ1314.42515 (Feretti et al. 2005), A 3376 (Bagchi et al. 2006), A 1240 and A 2345 (Bonafede et al. 2009a). Radio relics are likely energized by shock waves occurring during the cluster mergers, as confirmed by observational results obtained by e.g. Solovyeva et al. 2008, and Finoguenov et al. 2010.

Diffuse radio emission is also detected beyond clusters, on very large scales. An example is represented by the filament of galaxies $\mathrm{ZwCl} 2341.1+0000$ at $\mathrm{z} \sim 0.3$, first detected by Bagchi et al. 2002, and fecently confirmed by Giovannini et al. 2010, who also detect polarized emission. Noticeable are the complex radio structures detected on large scale at more than $2 \mathrm{Mpc}$ from the center of A 2255 (Pizzo et al. 2008), wich have been suggested to be connected with large scale structure formation shocks.

\section{Radio - X-Ray interaction in cooling core clusters}

The diffuse radio sources which may be detected at the center of cooling core clusters are classified as mini-halos, since they are morphologically similar to giant halos associated with merging clusters, but they are smaller in size (a few hundreds kpc). Although these sources are generally surrounding a powerful central radio galaxy, it has been argued that the energetics necessary to their maintenance is not supplied by the radio galaxy itself, but the electrons are reaccelerated by MHD turbulence in the cooling core region (Gitti et al. 2002). A peculiar case is represented by the cluster RXJ 1347.5-1145, one of the highest X-ray luminous clusters known so far. It is a relaxed clusters hosting a mini-halo, but it is also characterized by minor mergers in the cluster periphery. The

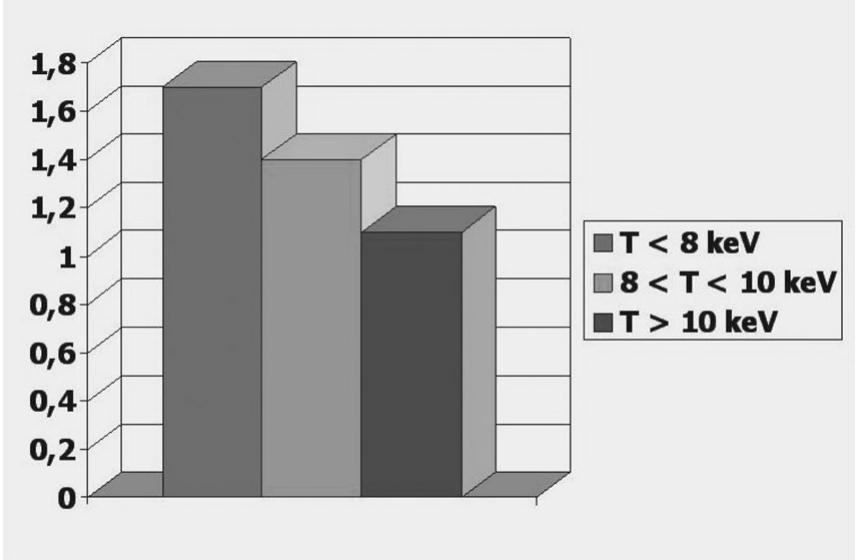

Figure 3. Average spectral index of radio halos for clusters in different ranges of temperature, showing that hotter clusters tend to host halos with flatter spectra. 
mini-halo, detected by Gitti et al. 2007, shows an asymmetrical structure, with elongation coincident with a X-ray subclump, suggesting that additional energy for the electron re-acceleration might be provided by the sub-merger event.

In some cooling core clusters, as A 13, A 85, A 133, A 4038, diffuse radio sources offset from the cluster center have been detected (Slee et al. 2001). They can be classified as mini relics, because of their small-intermediate size. They are characterized by very steep spectra $(\alpha \gtrsim 2)$, strong polarization, and filamentary structure when observed with sufficient resolution. The phenomenology of diffuse mini halos and mini relics, may be related to the radio source/ISM interaction in the central regions of cooling core clusters.

A spectacular example of the interaction between radio sources and the hot intracluster medium is represented by cavities in the X-ray gas distribution, filled with radio plasma. The first case has been detected by Böhringer et al. 1993 in the ROSAT image of NGC 1275 (3C 84) in the Perseus cluster. Here the thermal plasma is displaced by the inner parts of the radio lobes, causing a significant decrease of the X-ray surface brightness in those regions. The high spatial resolution of the Chandra X-ray Observatory has allowed the detection of X-ray cavities in the inner region of many cooling core clusters. These systems have been extensively studied in recent years, and a clear correspondence between regions of radio emission and deficits in the X-ray has been found in several cases (see Blanton et al. 2010 and references therein).
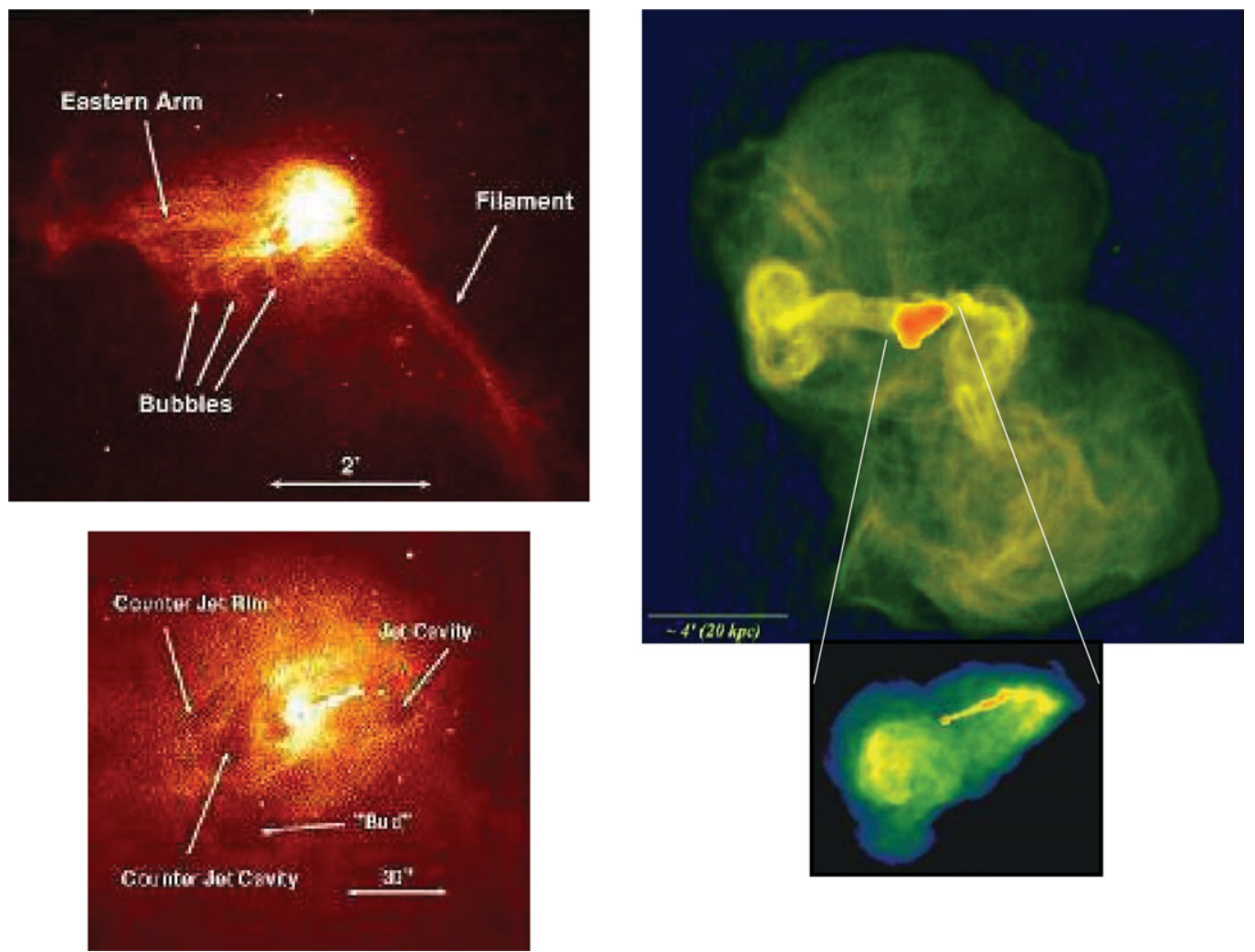

Figure 4. X-ray and radio structures of $\mathrm{M} 87$, a buoyant bubble in the center of a cool core cluster. The 2 left panels show the X-ray filaments and cavities detected with Chandra (Forman et al. 2007). The right panels show the large scale radio bubbles, with the radio jets in the inset (Owen et al. 2000). The angular scale is indicated within each image by a bar, which corresponds to $2^{\prime}$ in the top-left panel, to $30^{\prime \prime}$ in the bottom-left panel, and $4^{\prime}$ in the top-right panel. 
The interaction between the AGN jets and the ICM is believed to be the primary feedback mechanism between the AGN driven by supermassive black holes and its environment. Cooling of the hot intracluster medium in cluster centers can feed the supermassive black holes found in the nuclei of the dominant cluster galaxies, leading to AGN outbursts, which can reheat the gas. The relativistic radio jets of the associated radio sources are creating cavities, through one strong episode or several small episodes of energy release. AGN heating can come in the form of shocks, and buoyantly rising bubbles that have been inflated by radio lobes (Best et al. 2007, Birzan et al. 2008).

Recently, Falceta-Gonçalvez et al. 2010 used hydrodynamical simulations to study the structure and formation of the inflated cavities of NGC 1275, in the Perseus cluster, as due to the interaction between precessing jets and the warm intracluster plasma. They show that precession can create multiple pairs of bubbles, which rise and move away from the cluster core, and may therefore be related to the extended diffuse radio emission.

Another well studied radio source is M87 (Owen et al. 2000) in the nearby Virgo cluster (Fig. 4), where Forman et al. 2007 show X-ray structure and filaments which are related to the radio emission. Radio bubbles, blown into the cluster gas, rise buoyantly, expand and give rise to complex structures. Radio jets are confined within cavities at early times, but at later times the cosmic rays diffuse into the surrounding hot gas to form radio lobes with sizes much larger than the cavities. According to Mathews \& Brighenti 2008, the bubbles may eventually expand to the cluster outskirts, where the cosmic rays would impact into the surrounding medium, giving rise to mini relics.

\section{Magnetic fields in clusters}

The presence of diffuse radio sources in clusters demonstrates the existence of magnetic fields in the intracluster medium. An indipendent way to obtain information on the cluster magnetic field is from the analysis of Faraday Rotation. This kind of studies has allowed a breakthrough in the knowledge of the strength and structure of magnetic fields in clusters of galaxies, by analyzing the Rotation Measure of sources seen through the magnetized cluster medium (see e.g. Govoni \& Feretti 2004). Most recent studies are those on the Coma Cluster (Bonafede et al. 2010a), A 665 (Vacca et al. 2010) and the radio galaxy 3C 449 (Guidetti et al. 2010). The results obtained so far can be summarized as follows (see also Bonafede et al. 2010b): (i) magnetic fields are present in all clusters; (ii) at the center of clusters undergoing merger activity the field strenght is around $1 \mu \mathrm{G}$, whereas at the center of relaxed cooling core clusters the intensity is much higher $(\sim 10 \mu \mathrm{G})$; (iii) a model involving a single magnetic field coherence scale is not suitable to describe the observational data, because of different scales of field ordering and tangling.

Assuming a magnetic field power spectrum: $\left|B_{\kappa}\right|^{2} \propto \kappa^{-n}$ (Murgia et al. 2004), the range of spatial scales is found between $30-500 \mathrm{kpc}$ and the spectral index $n$ is in the range $2-4$. In A 2255, Govoni et al. 2006, from the comparison between the observations and the simulation of both the radio halo and the polarization of radio galaxies, obtain a flatter power spectrum at the center, and a steeper power spectrum at the periphery. This could originate from the different turbulence development in central and peripheral cluster regions.

The cluster magnetic field intensity shows a radial decline linked to the thermal gas density $\mathrm{n}_{e}$ as $B \propto \mathrm{n}_{e}^{x}$. A trend with $x=1 / 2$ is expected if the B field energy scales as the thermal energy, while $x=2 / 3$ if the $\mathrm{B}$ field results from a frozen-in field during the cluster collapse. The values of $x$ derived so far are in the range $0.5-1$, thus not conclusive. 


\section{Summary and future prospects}

The presence of relativistic plasma in clusters of galaxies is demonstrated by the existence of halos and relics detected in merging clusters, and the mini-halos and mini relics in cooling core clusters. These features, which were detected in the past only in a few clusters, are now becoming more and more common, in particular, it is remarkable that diffuse structures, on size scales of hundreds kpc are detected in low density environments. Diffuse radio emission is also detected in filaments and in the large scale structure. $\mathrm{X}$-ray cavities filled by radio bubbles are found at the center of cooling core clusters and represent the extreme example of interaction between the thermal and relativistic plasma.

Future prospects for the study of new radio sources of the different classes, in particular at low powers and at large redshifts, and study of the polarization come from the new generation radio telescopes, like the eVLA, LOFAR, the SKA precursors ASKAP and MeerKAT, and finally SKA. The study of relics and large scale diffuse sources is promising to trace the large scale structure formation and the cosmic web. Observations in the X-ray and optical domain are required to establish the cluster conditions, the merger evolutionary stage, the presence and properties of shocks, and the signatures of cluster turbulence. The comparison between the observational results and the theoretical expectations will be crucial to understand the physical link between the relativstic and thermal plasma.

\section{Acknowledgements}

L.F. wishes to thank the conference organizers for the invitation to this very stimulating meeting.

\section{References}

Bagchi, J., Enßlin, T. A., Miniati, F., Stalin, C. S., Singh, M., Raychaudhury, S., \& Humeshkar, N. B., 2002, NewA 7, 249

Bagchi, J., Durret, F., Lima Neto, G. B., \& Paul, S., 2006, Sci 314, 791

Best, P. N., von der Linden, A., Kauffmann, G., Heckman, T. M., \& Kaiser, C.R., 2007, MNRAS 379,894

Birzan, L., McNamara, B. R., Nulsen, P. E. J., Carilli, C.,L., \& Wise, M. W., 2008, ApJ 686, 859

Blanton, E. L., Clarke, T. E., Sarazin, C. L., Randall, S. W., \& McNamara, B. R., 2010, Publications of the National Academy of Sciences, Vol. 107, p. 7174

Böhringer, H., Voges, W., Fabian, A. C., Edge, A. C., \& Neumann, D. M., 1993, MNRAS 264, L25

Bonafede, A., Giovannini, G., Feretti, L., Govoni, F., \& Murgia, M., 2009a, A $\& A$ 494, 429

Bonafede, A., Feretti, L., Giovannini, G., Govoni, F., Murgia, M., Taylor, G. B., Ebeling, H., Allen, S., Gentile, G., \& Pihlström, Y., 2009b, A\&\&A 503, 707

Bonafede, A., Feretti, L., Murgia, M., Govoni, F., Giovannini, G., Dallacasa, D., Dolag, K., \& Taylor, G. B., 2010a, A\&3A 513, 30

Bonafede, A., Feretti, L., Murgia, M., Govoni, F., Giovannini, G., \& Vacca, V., 2010b, $A \mathscr{E} A$ in press, arXiv1009.1233

Brown, S. \& Rudnick, L., 2009, AJ 137, 3158

Cassano R., Brunetti G., \& Setti G., 2006 MNRAS 369, 1577

Fabian, A. C., 1994 ARA $\& A$ A2, 277

Falceta-Gonçalves, D., Caproni, A., Abraham, Z., Teixeira, D. M., \& de Gouveia Dal Pino, E. M., 2010 ApJ 713, L74

Feretti, L., Brunetti, G., Giovannini, G., Kassim, N., Orrú, E., \& Setti, G., 2004, JKAS 37, 315

Feretti, L., Schücker, P., Böhringer, H., Govoni, F., \& Giovannini, G., 2005, A $\mathscr{E} A$ 444, 157 
Feretti, L. \& Giovannini, G., 2008, in A Pan-Chromatic View of Clusters of Galaxies and the Large-Scale Structure, M. Plionis, O. Lopez-Cruz \& D. Hughes Eds., Lecture Notes in Physics, Vol. 740, p. 474

Finoguenov, A., Sarazin, C. L., Nakazawa, K., Wik, D. R., \& Clarke, T. E., 2010, ApJ 715, 1143

Forman, W., Jones, C., Churazov, E., Markevitch, M., Nulsen, P., Vikhlinin, A., Begelman, M., Böhringer, H., Eilek, J., Heinz, S., Kraft, R., Owen, F., \& Pahre, M., 2007, ApJ, 665, 1057

Giacintucci, S., Venturi, T., Cassano, R., Dallacasa, D., \& Brunetti, G., 2009, ApJ 704, L54

Giovannini, G. \& Feretti, L., 2004, JKAS 37, 323

Giovannini, G., Bonafede, A., Feretti, L., Govoni, F., Murgia, M., Ferrari, F., \& Monti, G., 2009, A\&A 507, 1257

Giovannini, G., Bonafede, A., Feretti, L., Govoni, F., \& Murgia, M., 2010, A\& $A$ 511, L5

Gitti, M., Brunetti, G., \& Setti, G., 2002, A\&A 386, 456

Gitti, M., Ferrari, C., Domainko, W., Feretti, L., \& Schindler, S., 2007, A\& A 470, L25

Govoni, F. \& Feretti, L., 2004, Int. J. Mod. Phys. D, Vol., 13, 1549

Govoni, F., Murgia, M., Feretti, L., Giovannini, G., Dolag, K., \& Taylor, G. B., 2006, A\& $A 460$, 425

Guidetti, D., Laing, R. A., Murgia, M., Govoni, F., Gregorini, L., \& Parma, P., 2010, A\&A 514, 50

Large, M. I., Mathewson, D. S., \& Haslam, C. G. T., 1959, Nat 183, 1663

Mathews, W. G. \& Brighenti, F., 2008, ApJ 676, 880

Murgia, M., Govoni, F., \& Feretti, L. , 2004, A\& $A$ 424, 429

Murgia, M., Govoni, F., Feretti, L., \& Giovannini, G., 2010, A\&A 509, 86

Owen, F. N., Eilek, J. A., \& Kassim, N. E., 2000, ApJ, 543, 611

Pizzo, R. F., de Bruyn, A. G., Feretti, L., \& Govoni, F., 2008, A\&A 481, L91

Röttgering, H. J. A., Wieringa, M. H., Hunstead, R. W., \& Ekers, R. D., 1977, MNRAS 290, 577

Slee, O. B., Roy, A. L., Murgia, M., Andernach, H., \& Ehle, M., 2001, AJ 122, 1172

Solovyeva, L., Anokhin, S., Feretti, L., Sauvageot, J. L., Teyssier, R., Giovannini, G., Govoni, F., \& Neumann, D., 2008, A\&A 484, 621

Vacca, V., Murgia, M., Govoni, F., Feretti, L., Giovannini, G., Orrú E., \& Bonafede, A., 2010, $A \mathscr{E} A 514,71$

van Weeren, R. J., Röttgering, H. J. A., Brüggen, M., \& Cohen, A., 2009, A $6 A$ 5 505, 991

van Weeren, R. J., Röttgering, H. J. A., Brüggen, M., \& Hoeft, M., 2010, Sci 330, 347 